\title{
Safety and efficacy of surgical treatments for axillary osmidrosis: a retrospective cohort study comparing conventional open excision with cartilage-shaver closed curettage
}

\author{
Ryutaro Tanaka, Daichi Morioka, Syuryo Akamine, Takafumi Shimizu, Koichi Kadomatsu \\ Department of Plastic Surgery, Showa University, Tokyo 142-8666, Japan.
}

Correspondence to: Prof. Daichi Morioka, Department of Plastic Surgery, Showa University, 1-5-8 Hatanodai, Shinagawa-ku, Tokyo 142-8666, Japan. E-mail: dmorioka@gmail.com

How to cite this article: Tanaka R, Morioka D, Akamine S, Shimizu T, Kadomatsu K. Safety and efficacy of surgical treatments for axillary osmidrosis: a retrospective cohort study comparing conventional open excision with cartilage-shaver closed curettage. Plast Aesthet Res 2020;7:17. http://dx.doi.org/10.20517/2347-9264.2020.12

Received: 21 Jan 2020 First Decision: 6 Mar 2020 Revised: 14 Mar 2020 Accepted: 2 Apr 2020 Published: 10 Apr 2020

Science Editor: Raúl González-García Copy Editor: Jing-Wen Zhang Production Editor: Jing Yu

\begin{abstract}
Aim: The aim of this study was to evaluate the usefulness of suction-assisted cartilage shaver (SACS) system closed curettage by comparing it with open excision regarding safety and efficacy.

Methods: A retrospective chart review was conducted for patients with axillary osmidrosis (AO) who underwent either open excision or SACS closed curettage between 2006 and 2018. We investigated the demographic data of patients and compared the postoperative complications and outcomes of the patients undergoing the two procedures.
\end{abstract}

Results: A total of 91 patients underwent SACS closed curettage and 188 patients underwent open excision. The complication rate in the SACS group (10.4\%) was significantly lower than that in the open excision group (20.7\%). Each procedure led to unsuccessful outcomes for two patients.

Conclusion: SACS closed curettage was safer than open excision for AO. Both procedures were extremely effective. Although decision-making for surgical treatment options for $\mathrm{AO}$ is affected by such other factors as discomfort in dressing, recovery time, scar formation, and cost, our results should be helpful for both surgeons and patients.

Keywords: Axillary osmidrosis, retrospective chart review, open excision, suction-assisted cartilage shaver system

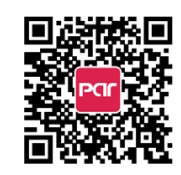




\section{INTRODUCTION}

Axillary osmidrosis (AO) is characterized by an offensive odor resulting from bacterial interaction with excessive apocrine secretions ${ }^{[1]}$. Especially in East Asian societies, AO impairs a patient's psychosocial functioning because of malodor and unsightly yellowish staining of clothing ${ }^{[1]}$.

Various nonsurgical and surgical treatments have been reported for AO. Nonsurgical treatments include the use of topical deodorant, subcutaneous injection of botulinum toxin- $\mathrm{A}$, and endoscopic thoracic sympathectomy. Surgical treatments include laser technology, axillary skin excision, suction curettage, and conventional open excision of apocrine glands $s^{[2-5]}$.

In general, less invasive treatments such as lasers have low complication rates, but are less effective. More invasive treatments such as open surgery are more effective and have a lower recurrence rate, but they have a higher complication rate ${ }^{[2]}$.

Inaba and $\mathrm{Ezaki}^{\left[{ }^{[6]}\right.}$ reported that surgical treatments have been traditionally performed for patients with AO in Japan since the 1950s. Since open excision of axillary sweat glands was first reported as a surgical treatment for hyperhidrosis by Skoog and Thyresson ${ }^{[7]}$ in 1962, many surgeons and dermatologists have performed similar procedures for AO. Various modifications on types of incision, drainage methods, suture techniques, and tie-over dressings have been attempted ${ }^{[1,8,9]}$. Open excision is still commonly performed in Asian countries because the entire procedure can be completed using a basic set of surgical instruments that include forceps, skin hooks, dissecting scissors, and suture materials. It is also cost effective, compared with other treatment options ${ }^{[1,10]}$.

The suction-curettage approach for AO was first introduced by the Taiwanese surgeons Ou et al. ${ }^{[11]}$ in 1998. It has been subsequently modified by many Asian surgeons and dermatologists. The modifications include a curette provided with a vacuum system ${ }^{[12]}$, Fatemi/Cassio cannula ${ }^{[13]}$, and a suction-assisted cartilage shaver (SACS) system ${ }^{[14,15]}$. To date, subdermal excision as an open surgical procedure and suction curettage as a closed surgical procedure are the two major approaches for AO.

While other suction-curettage techniques manually remove sweat glands, SACS uses a double-sheathed electric oscillating cutter to aggressively remove sweat glands. The effectiveness of SACS closed curettage was reported to be comparable to that of open excision ${ }^{[14-20]}$. Shin et al. ${ }^{[21]}$ recently performed a metaanalysis of the safety and efficacy of closed surgery compared with open surgery for AO and concluded that suction curettage was safer than open surgery, but less effective than open surgery. Their analysis evaluated more than one type of closed surgical procedure, as described in the previous paragraph, and open surgery included not only conventional techniques but also en bloc skin excision and endoscopic surgery. To our knowledge, no studies in the English-language literature comparing the safety and efficacy of SACS closed curettage versus conventional open excision have been published.

Since 2006, we have used, based on the patient's choice, either SACS closed curettage or open surgery to treat more than 600 patients with $\mathrm{AO}^{[1]}$. Our impression has been that SACS is preferable to open excision regarding its safety and efficacy. The aim of this retrospective cohort study was to determine whether our impression of the usefulness of SACS was accurate based on statistical analysis compared with conventional open surgery.

\section{METHODS}

\section{Patients}

The Showa University Hospital institutional research board approved this study (approval No. 2928). A retrospective chart review was conducted for consecutive patients with $\mathrm{AO}$ who underwent either open 
excision or SACS closed curettage at Showa University Hospital or affiliated private clinics. Patients who had previously undergone other surgical treatments for AO were excluded from this study. Prior to surgery, the odor level of each patient was determined by a previously described gauze test ${ }^{[1]}$. The scale ranged from Level 1 (no odor) to Level 5 (severe odor), and patients with Levels 3 (moderate) to 5 were considered suitable for surgical treatments. The treatment option was chosen by each patient and his/her family instead of by the operating surgeon. Surgical procedures for more than $90 \%$ of patients were performed by a single surgeon (D.M.), and the other procedures were performed by surgeons under his supervision.

\section{SACS system closed curettage}

Patients lay in the supine position, with their arms abducted $90^{\circ}-120^{\circ}$. The region of hair-bearing skin plus a 5-mm margin was delineated and 30-40 mL of a solution of $0.25 \%$ lidocaine with 1:200,000 epinephrine was injected for local anesthesia. A 5-mm skin incision was made at either the medial or lateral edge of the marked skin. Suction of the marked area was first performed by a conventional liposuction cannula (3-mm diameter) inserted through the incision, and the subdermal layer containing the sweat glands (mainly apocrine glands) was removed by the SACS system (TPS; Stryker, Kalamazoo, MI, USA), equipped with an electric shaver (Formula; Stryker). This system is normally used for endoscopic arthroplasty in orthopedic surgery. It is composed of an outer cannula equipped with a grid that safely removes apocrine glands, and an inner cannula equipped with a serrated blade that strongly oscillates to remove apocrine glands [Figure 1]. The shaver was also connected to a vacuum pump (S200; Kakinuma Medical Inc., Tokyo, Japan) by a suction tube, which immediately removed the tissues containing the apocrine glands and discarded them into a container. After the subdermal tissues of the entire marked area were evenly excised, 2-6 quilting sutures (depending on the size of operated area) were used to anchor the loosened skin to the underlying fascia, and a drainage tube was inserted to extend through the skin incision. Compression by tie-over dressings and elastic bandages was applied for 3-5 days postoperatively. Anchoring sutures and sutures closing the incision were removed one week after surgery. The patient was allowed to perform full rangeof-motion of the shoulder two weeks postoperatively.

\section{Open excision}

The operating position, local anesthesia, and operating area for conventional open excision were the same as for patients undergoing SACS curettage. According to a previously reported technique ${ }^{[22]}$, a small elliptical section of skin (3-6 cm long) in the center of the hair-bearing region was removed. The axillary skin was undermined from the incision both medially and laterally above the fascia. A skin hook was used to turn over the undermined skin, and the layer mainly containing the apocrine glands was identified [Figure 2]. Subdermal tissues containing the apocrine glands were pruned evenly from the center up to the edge of the marked area by dissecting scissors, and bleeding was controlled by electrocauterization. Following wound closure with 5/0 nylon sutures, 2-6 quilting sutures were used to anchor the skin flap to the underlying fascia, and a drainage tube was inserted to extend from the edge of the incision. Compression was achieved using tie-over dressings and elastic bandages, which were applied for 5-7 days postoperatively. Quilting sutures were removed one week after surgery, and incision sutures were removed between two and three weeks after surgery. The patient was usually allowed to perform full range-of-motion of the shoulder four weeks postoperatively.

\section{Data assessments}

Safety was assessed according to the occurrence of acute moderate-to-severe adverse events, which included hematoma, seroma, and delayed wound healing. The events were assessed for each axilla. When more than two adverse events occurred in the same axilla (e.g., skin necrosis plus wound infection), the major event was used to determine the overall rate of occurrence for patients undergoing either closed or open surgery. Mild adverse events such as erosion, contact dermatitis due to dressing tape, and transient erythema were excluded from the assessment. 

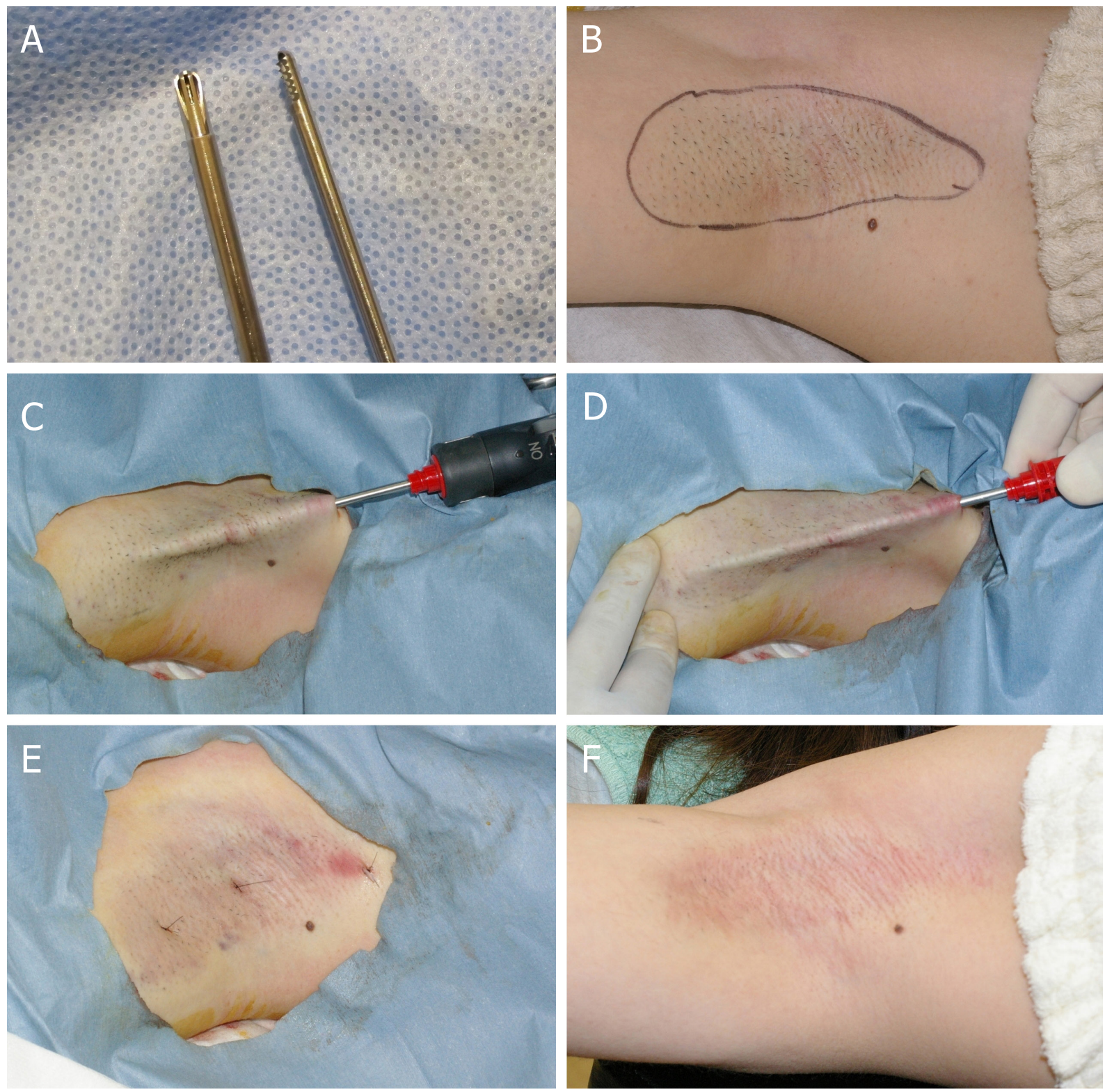

Figure 1. Closed-curettage surgery using the suction-assisted cartilage shaver system. A: the shaver is composed of an outer cannula with grid (left) and serrated inner cannula (right); B: the operating area is hair-bearing skin with a 5-mm margin; C: following subdermal undermining, the shaver is inserted from the edge of the marked region; D: the axillary skin is so thin that the shaver can be seen through the skin; E: the wound is closed with nylon sutures after insertion of a drainage tube, and two quilting sutures were made in this case; F: ten-month postoperative photograph. Note that axillary hair appears to have been almost permanently removed

The efficacy of each procedure was assessed by determining the rate of recurrence, which was mainly based on the patient's self-reported complaint or occasionally assessed by a physician performing the gauze test. The recurrence rate included overall assessments such as "not improved" or "ineffective", as well as "recurrence". The complication and recurrence rates of the patients who underwent SACS closed curettage were compared with those rates of the patients who underwent open excision.

Patients followed-up postoperatively for $\geq 3$ months were included in this survey. Some patients graded the level of their subjective satisfaction for the entire treatment program (factors including surgical procedures, postoperative pain, discomfort, outcome, scar appearance, and cost) with a visual analog scale ranging from 0 (not satisfied) to 10 (fully satisfied). 

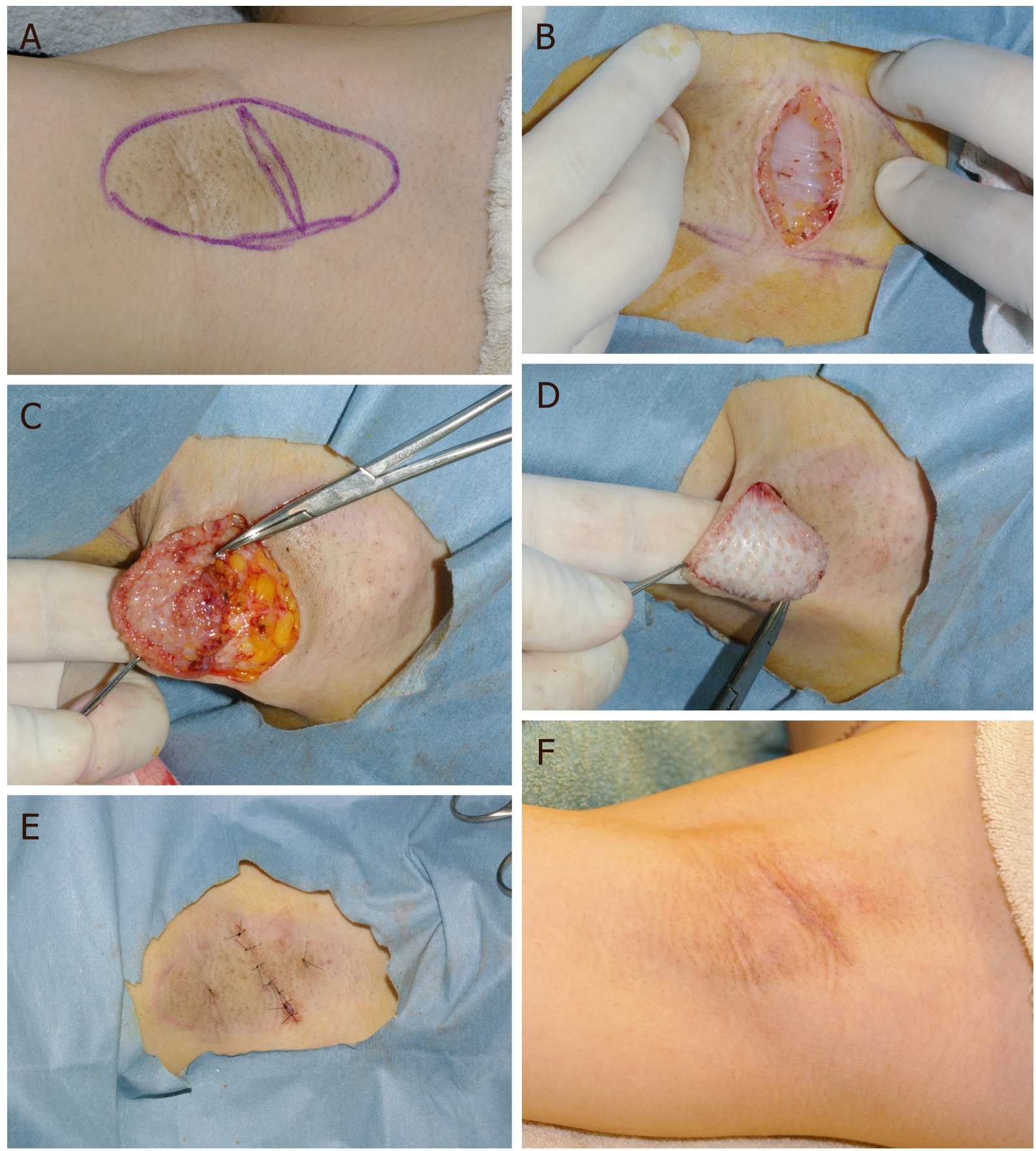

Figure 2. Open excision procedures: A: the incision plan according to Rigg ${ }^{[22]}$; B: a small elliptical sliver of skin is removed, and the apocrine glands lined above the fascia are seen; C: after the subdermal layer of the axilla is undermined, the apocrine gland layer is seen; D: the apocrine gland layer is completely pruned, leaving the sebaceous glands; E: the wound is closed by $5 / 0$ nylon sutures; two quilting sutures were made in this case. A drainage tube is inserted from the lower edge of incision wound; F: six-month postoperative photograph

\section{Statistical analysis}

The mean ages of the two patient groups were compared by the Mann-Whitney $U$-test. Other categorical variables were compared by the chi-squared test. When the expected values were too small to be analyzed by the chi-squared test, the Fisher exact test or Yates continuity correction was used for the comparison. Statistical analysis was performed by Microsoft Excel 2010 (Microsoft, Redmond, WA, USA). $P<0.05$ was considered statistically significant. 
Table 1. Patient characteristics and postoperative outcomes of closed curettage by the SACS and open excision for axillary osmidrosis

\begin{tabular}{llll}
\hline Procedure & SACS & Open excision & P \\
\hline Number (male:female), $n$ & $91(21: 70)$ & $168(56: 112)$ & 0.09 \\
Mean age \pm SD, $\mathrm{m}$ & $28.6 \pm 7.6$ & $28.0 \pm 7.3$ & 0.51 \\
Odor levels, $n(\%)^{\star}$ & & & 0.06 \\
3 & $12(13.2)$ & $42(25.0)$ & \\
4 & $53(58.2)$ & $90(53.7)$ & \\
5 & $26(28.6)$ & $36(21.3)$ & 0.002 \\
Total complications, $n(\%)^{\star *}$ & $19(10.4)$ & $70(20.8)$ & 0.06 \\
Hematoma & $9(4.9)$ & $34(10.1)$ & $<0.001$ \\
Wound dehiscence & $0(0.0)$ & $21(6.3)$ & 0.09 \\
Skin necrosis & $3(1.6)$ & $11(3.3)$ & 0.02 \\
Seroma & $5(2.7)$ & $1(0.3)$ & 0.28 \\
Others & $2(1.1)$ & $2(0.6)$ & 0.61 \\
Recurrence, $n$ (\%) & $2(2.2)$ & $2(1.2)$ & 0.10 \\
Mean patient satisfaction & $8.8(n=78)$ & \\
\hline
\end{tabular}

*Odor levels 1 (no odor) and 2 (faint odor) were not indicative for surgery; ${ }^{* *}$ complications were assessed by axilla ( $n=182$ for SACS, $n$ $=336$ for open excision groups); ${ }^{\star \star \star}$ other rare events included infection, sensory disturbance, and arterial injury; ${ }^{\star \star \star \star}$ patient satisfaction was not assessed for all patients ( $n=48$ for SACS, $n=78$ for open excision groups). SACS: suction-assisted cartilage shaver system

\section{RESULTS}

\section{Patient characteristics}

The characteristics of the patients are summarized in Table 1. A total of 188 patients underwent open excision and 91 patients underwent closed curettage by the SACS system. The male/female ratio, mean age, and odor levels were similar between the SACS and open-surgery patients. The duration of postoperative follow-up was 5.2 months (minimum 3 months, maximum 64 months), and the follow-up periods were statistically similar between the two patient groups.

\section{Safety and efficacy of the procedures}

The complication rate of the patients treated by the SACS system was significantly lower than the complication rate of the patients undergoing open surgery $(10.4 \% v s .20 .7 \% ; P=0.002)$. Table 1 shows detailed comparisons, and Figure 3 shows examples of the complications. The most frequent complication in either group was hematoma [Figure $3 \mathrm{~A}$ ], with no significant difference between the rates $(P=0.06)$. Wound dehiscence [Figure 3B] was seen only in patients undergoing open surgery. Delayed wound healing including skin necrosis and wound dehiscence was more frequently seen in patients undergoing open surgery $(P<0.001)$. Seroma [Figure $3 \mathrm{C}$ ] was significantly more frequent in the patients treated by the SACS system $(10.4 \%, P=0.002)$.

In each treatment group, two patients had a recurrence or thought the outcome was unsuccessful, representing $1.1 \%$ of patients undergoing SACS closed curettage and $2.2 \%$ of patients undergoing open surgery. The difference was not significant $(P=0.614)$. Patient satisfaction for both procedures was very high (8.5/10 for SACS $v$ s. 8.8/10 for open excision), with no significant difference $(P=0.010)$.

\section{DISCUSSION}

\section{Previously reported cohorts}

Several retrospective cohort studies have reported the treatment options for AO. For example, Park and Shin ${ }^{[23]}$ compared conventional open excision, liposuction, laser vaporization, and ultrasonic aspiration, and they concluded that the best option was open excision. Chen et al. ${ }^{[24]}$ compared laser coagulation and suction curettage, and concluded that suction curettage produced a higher incidence of complications than laser coagulation, but was more effective than laser coagulation. Yang et al. ${ }^{[5]}$ recently compared 

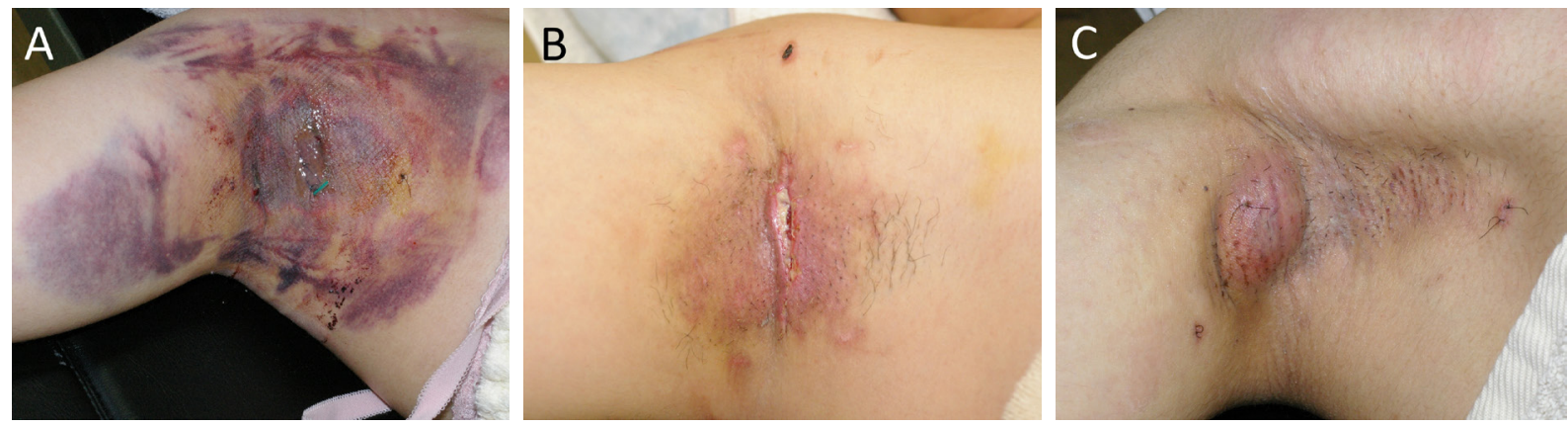

Figure 3. Examples of postoperative complications: A: hematoma after open excision; B: wound dehiscence after open excision; and C: seroma after closed curettage by the suction-assisted cartilage shaver system

the outcomes of microwave coagulation, suction curettage, and laser coagulation. They concluded that microwave coagulation and suction curettage were superior to laser coagulation, but the complication rate of suction curettage was much higher than the rates of the other two procedures. In the English-language literature, however, no studies have compared the two major surgical treatments (suction curettage and conventional open surgery). We believe that our study was meaningful because we compared the outcomes of these two procedures, $90 \%$ of which were performed by a single surgeon.

\section{SACS system}

To our knowledge, only one comparative observation of the efficacy of curettage by the SACS system versus $\mathrm{AO}$ treatment by open excision has been published in Japanese ${ }^{[15]}$. The seven other English-language reports on $\mathrm{AO}$ treatment by closed-curettage $\mathrm{SACS}^{[2,14,16-20]}$ were uncontrolled clinical observations or case series, as listed in Table 2. During early SACS procedures, the tip of the outer cannula was open, which often accidentally perforated the axillary skin during the procedure ${ }^{[14,15]}$. Therefore, curettage by SACS was performed under endoscopy. Later, most surgeons modified the procedure and used an outer cannula tip equipped with a grid, so that the apocrine glands could be safely removed while skin perforation and damage to the subdermal plexus were avoided [Figure 1A]. There are two different types of inner cannula tips, consisting of smooth or serrated blades. Most surgeons, including our group, have preferred the latter type of tip, because it more thoroughly removes the apocrine glands.

As shown in Table 2, the complication rate for the SACS system in our study (10.4\%) was relatively higher than the rate in previous studies (0\%-7.7\%). The higher rate might be because we extensively undermined the hair-bearing skin plus a 5-mm margin to remove the apocrine glands thoroughly. A few authors spared fibrous cords and perforating vessels during curettage by the SACS system. Chern et al. ${ }^{[18]}$ preserved fibrovascular bands and found a single adverse event involving 1 of 60 axillae. There were no recurrences. They found, however, that the mean efficacy rate was relatively low, since excellent results were obtained from only $67 \%$ of patients. Similarly, Hsu and Wang ${ }^{[19]}$ preserved the subcutaneous fibrous septa, and did not observe any adverse events. However, 3 of 19 (15.8\%) patients developed recurrence and underwent revision surgery. We concluded that the greater is the number of preserved fibrovascular bands, the greater is the number of remaining apocrine glands around the bands.

\section{Complications}

Among the acute adverse events seen in this study, only the incidence of seroma in the patients undergoing SACS closed curettage was higher than the incidence in the patients undergoing open surgery (2.7\% vs. $0.3 \%)$. Interestingly, in patients undergoing abdominoplasty, the incidence of seromas in the patients undergoing abdominoplasty combined with closed liposuction is higher than in patients undergoing conventional open abdominoplasty ${ }^{[25]}$. A seroma results from the rupture of lymph vessels, but the associated factors 
Table 2. Reports on closed curettage by suction-assisted cartilage shaver system for axillary osmidrosis

\begin{tabular}{|c|c|c|c|c|c|c|}
\hline Ref. & $\begin{array}{c}\text { Patients } \\
\text { (male:female), } n\end{array}$ & Mean age, year & $\begin{array}{c}\text { Follow-up period, } \\
\text { month }\end{array}$ & $\begin{array}{c}\text { Complication, } n \\
(\%)\end{array}$ & $\begin{array}{c}\text { Recurrence, } n \\
(\%)\end{array}$ & $\begin{array}{c}\text { Type of shaver, inner/ } \\
\text { outer }\end{array}$ \\
\hline Tung $^{[14]}$ & $64(21: 43)$ & NR & $6-13$ & $5 / 128(3.9)^{\star}$ & $3 / 128(2.3)$ & Serrated/open \\
\hline Matsuda $^{[15]}$ & $77(20: 57)$ & 27 & $3-36$ & $7 / 154(4.5)^{\star}$ & $2 / 77(2.6)$ & Serrated/open + grid \\
\hline Lee et al. ${ }^{[2]}$ & $89(15: 74)$ & NR & $14-28$ & $0 / 89(0)$ & $0 / 89(0)$ & Smooth/grid \\
\hline$W u^{[16]}$ & $156(26: 130)$ & 23 & $6-59$ & $12 / 156(7.7)$ & $4 / 156(2.6)$ & Smooth/grid \\
\hline Huang et al. ${ }^{[17]}$ & $70(8: 62)$ & 26 & $6-35$ & NR & $1 / 70(1.4)$ & NR \\
\hline Chern et al. ${ }^{[18]}$ & $30(10: 20)$ & 22 & $3-13$ & $1 / 60(1.7)^{\star}$ & $0 / 30(0)$ & Smooth/grid \\
\hline Hsu and Wang ${ }^{[19]}$ & $19(4: 15)$ & 34 & 3 & $0 / 38(0)^{\star}$ & $3 / 19$ (15.8) & NR \\
\hline Tseng et $a{ }^{[20]}$ & $39(11: 28)$ & 26 & $12-69$ & $0 / 39(0)$ & $0 / 39(0)$ & NR \\
\hline Current study & $91(21: 70)$ & 28.2 & $3-60$ & $19 / 182(10.4)^{\star}$ & $2 / 91(2.2)$ & Serrated/grid \\
\hline
\end{tabular}

`Evaluation by axilla. NR: not reported

are thought to be multifactorial, including subdermal space, lymphatic drainage, the use of a cauterization device, and skin shearing ${ }^{[25]}$. Since the axillary region is well supplied with lymph vessels, we speculate that the mechanism involved in an axillary seroma is similar to that for an abdominal seroma. Seroma and hematoma in SACS closed-curettage procedures as well as in abdominoplasty might be avoided with an increased number of anchoring sutures and a longer drainage period. In fact, Tseng et al. ${ }^{[20]}$ created multiple drainage holes and quilting sutures following the use of a shaver and did not find any adverse events. However, such additional procedures resulted in unsightly scarring.

\section{Efficacy}

With regard to the assessment of efficacy, our study has some limitations. Postoperative efficacy was primarily assessed based on the patient's opinion, and complaints such as "recurrent odor", "not improved", or "not effective" were further reconfirmed by our gauze test. However, the perception of satisfaction varied based on the individual patient. Even for some patients who were satisfied with their procedure, recurrence might have been diagnosed by the gauze test. Second, although our minimum follow-up period was three months, which is similar to previously reported studies, the follow-up period might be too short. Wang et al. ${ }^{[4]}$ compared the efficacy of their suction-curettage procedure with that of conventional open surgery, and they concluded that an evaluation of the final outcomes at three months after surgery might be too early. In fact, several authors have reported patients with axillary odor that recurred six months or longer after their procedure $^{[1,5,15]}$. Thus, the recurrence rates in this study were possibly underestimated for both treatments.

\section{Emerging treatments}

Recently, emerging treatments for axillary hyperhidrosis, such as microwave and radiofrequency technologies, have been reported to have some positive effects for $\mathrm{AO}$ as well ${ }^{[2,5]}$. Although the procedures using these new technologies are more costly than suction curettage procedures, the procedures are nonsurgical and might provide permanent effects. Yang et al. ${ }^{[5]}$ performed a retrospective cohort study that compared microwave technology and suction curettage and found that the recurrence rate was higher with microwave than with suction curettage, although the complication rate after microwave treatment was much lower than the rate after suction curettage. Further comparative studies between novel technologies and SACS closed curettage are needed with respect to complications and recurrence, as well as regarding cost, postoperative discomfort, recovery time, and patient satisfaction.

In conclusion, the results of previous studies have suggested that suction-curettage techniques for AO are much safer than conventional open surgery, but are inferior regarding recurrence rate. This study found that SACS closed curettage was as safe as other suction-curettage techniques, and as effective as conventional open surgery. In addition, the periods of discomfort due to dressings and limited range of motion in patients undergoing SACS closed curettage were half as long compared to those periods 
in patients undergoing open surgery. Although further comparative studies are needed on emerging technologies versus SACS closed curettage, to date, SACS closed curettage should be considered an ideal surgical treatment for AO; however, decision-making regarding surgical treatment options for AO is affected by other factors, such as dressing discomfort, recovery time, scar formation, and cost. Our results should be helpful for both surgeons and patients.

\section{DECLARATIONS}

\section{Authors' contributions}

Made substantial contributions to conception and design of the study: Tanaka R, Morioka D, Akamine S, Kadomatsu K

Performed data analysis and interpretation: Tanaka R, Shimizu T

Performed data acquisition: Tanaka R, Morioka D

\section{Availability of data and materials}

Not applicable.

\section{Financial support and sponsorship}

None.

\section{Conflicts of interest}

All authors declared that there are no conflicts of interest.

\section{Ethical approval and consent to participate}

The Showa University Hospital institutional research board approved this study (approval no. 2928).

\section{Consent for publication}

Written consent was obtained for publication for patient images.

\section{Copyright}

(c) The Author(s) 2020.

\section{REFERENCES}

1. Morioka D, Ohkubo F, Amikura Y. Clinical features of axillary osmidrosis: a retrospective chart review of 723 Japanese patients. J Dermatol 2013;40:384-8.

2. Lee JC, Kuo HW, Chen CH, Juan WH, Hong HS, et al. Treatment for axillary osmidrosis with suction-assisted cartilage shaver. Br J Plast Surg 2005;58:223-7.

3. Morioka D, Nomura M, Lan L, Tanaka R, Kadomatsu K. Axillary osmidrosis: past, present, and future. Ann Plast Surg 2019; Epub ahead of print. doi: 10.1097/SAP.0000000000002111.

4. Wang $\mathrm{C}, \mathrm{Wu} \mathrm{H}, \mathrm{Du} \mathrm{F}, \mathrm{Le} \mathrm{S}$, Zheng S. Axillary osmidrosis treatment using an aggressive suction-curettage technique: a clinical study on paired control. Aesthet Plast Surg 2015;39:608-15.

5. Yang HH, Miao Y, Chen YT, Hu ZQ. Minimally invasive approaches to axillary osmidrosis treatment: a comparison between superficial liposuction with automatic shaver curettage, subcutaneous laser treatment, and microwave-based therapy with a modified technique. J Cosmet Dermatol 2018;18:594-601.

6. Inaba M, Ezaki T. New instrument for hircismus and hyperhidrosis operation: subcutaneous tissue shaver. Plast Reconstr Surg 1977;59:864-6.

7. Skoog T, Thyresson N. Hyperhidrosis of the axillae, a method of surgical treatment. Acta Chir Scand 1962;124:531-8.

8. Li ZR, Sun CW, Zhang JY, Qi YQ, Hu JZ. Excision of apocrine glands with preservation of axillary superficial fascia for the treatment of axillary bromhidrosis. Dermatol Surg 2015;41:640-4.

9. Dai Y, Xu AE, He J. A refined surgical treatment modality for bromhidrosis: Subcutaneous scissor with micropore. Dermatol Ther 2017;30:e12484.

10. Liu Q, Zhou Q, Song Y, Yang S, Zheng J, et al. Surgical subcision as a cost-effective and minimally invasive treatment for axillary 
osmidrosis. J Cosmet Dermatol 2010;9:44-9.

11. Ou LF, Yan RS, Chen IC, Tang YW. Treatment of axillary bromhidrosis with superficial liposuction. Plast Reconstr Surg 1998;102:1479-85.

12. Shi Z, Yan X, Ye X. Modified tumescent superficial suction with curettage treatment for axillary bromidrosis: clinical experience of 280 cases. Aesthet Plast Surg 2014;38:151-5.

13. Kim WO, Song Y, Kil HK, Yoon KB, Yoon DM. Suction-curettage with combination of two different cannulae in the treatment of axillary osmidrosis and hyperhidrosis. J Eur Acad Dermatol Venereol 2008;22:1083-8.

14. Tung TC. Endoscopic shaver with liposuction for treatment of axillary osmidrosis. Ann Plast Surg 2001;46:400-4.

15. Matsuda K. Surgical treatment of axillary osmidrosis with the shaver system. Jpn J Plast Surg 2004;47:1253-9. (in Japanese)

16. Wu WH. Ablation of apocrine glands with the use of a suction-assisted cartilage shaver for treatment of axillary osmidrosis. An analysis of 156 cases. Ann Plast Surg 2009;62:278-83.

17. Huang $\mathrm{YH}$, Yang $\mathrm{CH}$, Chen $\mathrm{YH}$, Chen $\mathrm{CH}$, Lee SH. Reduction in osmidrosis using a suction-assisted cartilage shaver improves the quality of life. Dermatol Surg 2010;36:1573-7.

18. Chern E, Yau D, Chuang FC, Wu WM. Arthroscopic shaver with refinement for axillary osmidrosis. Int J Dermatol 2010;49:813-7.

19. Hsu KC, Wang KY. Sparing subcutaneous septa avoids skin necrosis in the treatment of axillary bromhidrosis with suction-curettage shaving. J Cosmet Dermatol 2019;18:892-6.

20. Tseng YJ, Lee CH, Lin SH. Modified suction-assisted cartilage shaver for axillary osmidrosis. Biomed Res Int 2019;2019:7314753.

21. Shin JY, Roh SG, Lee NH, Yang KM. Osmidrosis treatment approaches. A systematic review and meta-analysis. Ann Plast Surg 2017;78:354-9.

22. Rigg BM. Axillary hyperhidrosis. Plast Reconstr Surg 1977;59:334-42.

23. Park YJ, Shin MS. What is the best method for treating osmidrosis? Ann Plast Surg 2001;47:303-9.

24. Chen YT, Shih PY, Chen HJ, Chen TJ. Treatment of axillary osmidrosis: a comparison between subcutaneous laser and superficial liposuction curettage. J Eur Acad Dermatol Venereol 2015;29:2019-23.

25. Vidal P, Berner JE, Will PA. Managing complications in abdominoplasty: a literature review. Arch Plast Surg 2017;44:457-8. 